\title{
Impact of renin-angiotensin system blocker after aortic valve replacement-a systematic review and meta-analysis
}

\author{
Li Zeng ${ }^{1 \#}$, Junli Li ${ }^{2 \#}$, Jinghan Yang $^{1}$, Yanbiao Liao ${ }^{2} \wedge$, Mao Chen ${ }^{2}$ \\ ${ }^{1}$ West China Hospital, Sichuan University, Chengdu, China; ${ }^{2}$ Department of Cardiology, West China Hospital, Chengdu, China \\ Contributions: (I) Conception and design: Y Liao, M Chen; (II) Administrative support: M Chen; (III) Provision of study materials or patients: Y Liao, \\ L Zeng; (IV) Collection and assembly of data: Y Liao, M Chen; (V) Data analysis and interpretation: L Zeng, J Li, Y Liao; (VI) Manuscript writing: \\ All authors; (VII) Final approval of manuscript: All authors. \\ "These authors contributed equally to this work. \\ Correspondence to: Yanbiao Liao, MD, PhD; Mao Chen, MD, PhD. Department of Cardiology, West China Hospital, Sichuan University, 37 Guoxue \\ St., Chengdu 610041, China. Email: ahlyb1990@163.com; hmaochen@vip.sina.com.
}

Background: Data reporting the impact of renin-angiotensin system inhibitor (RASi) after aortic valve replacement (AVR) is controversy.

Methods: The PubMed database was systematically searched for studies reporting the mortality and hazard ratios (HRs) of RASi following surgical and transcatheter AVR (SAVR, TAVR). Random-effects model was used when the $\mathrm{I}^{2}$ statistic was more than $50 \%$ and its $\mathrm{P}$ value was less than 0.05 , otherwise, the fixed-effects model was conducted.

Results: Nine articles incorporating 33,063 patients were eligible. Patients having the description of RASi were associated with lower all-cause mortality at 30 days (OR, 0.80, 95\% CI, 0.69 to 0.94), 1 year (OR, 0.75, 95\% CI, 0.69 to 0.81 ) and beyond 1 year (OR, 0.52, 95\% CI, 0.38 to 0.73 ) after AVR. Consistently, patients with RASi had lower risk for all-cause mortality (HR, 0.87, 95\% CI, 0.84 to 0.91 ) beyond 1 year following AVR albeit adjusting confounders. Interestingly, beneficial effect of RASi was still observed in patients with preserved ejection fraction following TAVR (HR, 0.90, 95\% CI, 0.87 to 0.94). In addition, patients taking RASi had lower cardiovascular mortality than those patients without RASi after TAVR (30 days, OR, 0.63, 95\% CI, 0.44 to 0.90 ; 1 year, OR, 0.60, $95 \%$ CI, 0.50 to 0.73 ; beyond 1 year, OR, $0.63,95 \%$ CI, 0.54 to 0.74 ).

Conclusion: Patients with RASi exhibited better short- and long-term survival following AVR compared to those patients without RASi, which warranted further studies to support such findings.

Keywords: Aortic valve replacement (AVR); transcatheter aortic valve replacement (TAVR); renin-angiotensin system inhibitor (RASi)

Submitted May 21, 2020. Accepted for publication Sep 02, 2020.

doi: 10.21037/apm-20-1155

View this article at: http://dx.doi.org/10.21037/apm-20-1155

\section{Introduction}

Aortic stenosis (AS) is a common valvular heart disease in the aging population, which is always associated with cardiac hypertrophy in response to the increased afterload (1). The activation of renin-angiotensin system (RAS) was considered to accelerate the left ventricular (LV) remodeling in patients with AS (2). Inhibition of RAS was demonstrated to decrease blood pressure, modulate myocardial hypertrophy and improve clinical outcomes in patients having heart failure with reduced left ventricular ejection fraction (LVEF), which made RAS inhibitors (RASi) to be widely used in patients with cardiovascular disease $(3,4)$. While, use of RASi including angiotensin-converting enzyme inhibitors (ACEIs)

\footnotetext{
$\wedge$ ORCID: 0000-0002-9853-0012.
} 


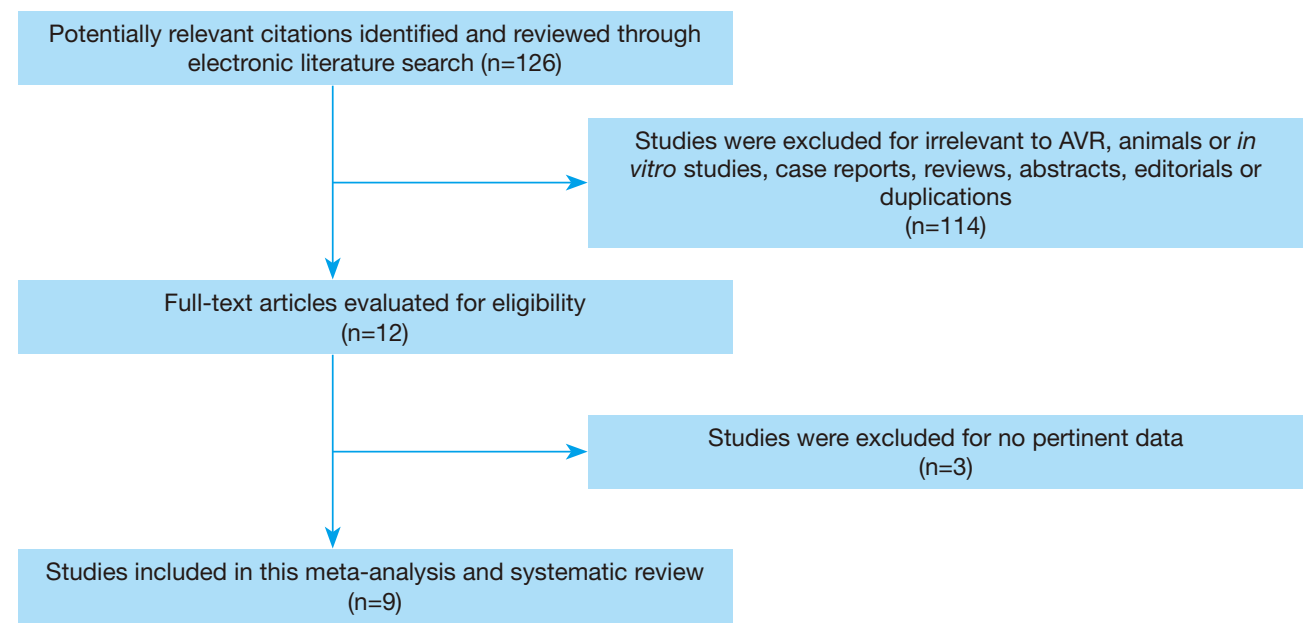

Figure 1 The process of study selection. AVR, aortic valve replacement.

and angiotensin II receptor blockers (ARBs) has traditionally been avoided in patients with AS, inasmuch as the concern of deteriorated hemodynamic results and subsequent hypotension (5). In fact, more increasing data demonstrate the safety and even potential beneficial effect of ACEIs/ARBs in patients with AS (6-9).

Aortic valve replacement (AVR) including surgical aortic valve replacement (SAVR) and transcatheter aortic valve replacement (TAVR), was an effective strategy to treating patients with AS. However, if patients with AS were not treated timely, excessive LV hypertrophy and fibrosis would compromise the clinical outcome after AVR $(10,11)$. Numerous data demonstrated that patients with persistence of LV hypertrophy and fibrosis were at high risk for increased long-term mortality after AVR $(12,13)$. However, there is no specific recommendation for the use of ACEIs/ARBs after AVR to attenuate LV hypertrophy and fibrosis, and debate surrounded the impact of ACEIs/ARBs on clinical outcome after AVR. Therefore, we performed the meta-analysis and systematic review to explore the effect of RASi on clinical outcome after AVR including SAVR and TAVR in patients with AS. We reported the present article in accordance with the Preferred Reporting Items for Systematic Reviews and Meta-Analyses (PRISMA) reporting checklist (available at http://dx.doi.org/10.21037/apm-20-1155).

\section{Methods}

\section{Data sources and study selection}

A literature search of the PubMed online database was conducted to identify studies reporting the impact of RASi on clinical outcome after AVR on February 9, 2020 (Figure 1). The electronic search terms were as follows: (renin-angiotensin system inhibitor OR ACEI OR ARB OR renin-angiotensin system OR ACE inhibitor OR renin-angiotensin system blockade) AND (surgical aortic valve OR SAVR OR transcatheter aortic valve OR aortic valve replacement OR AVR OR TAVR OR TAVI). Additionally, reference lists of pertinent articles were also screened manually in case of omitting potential relevant citations. All citations were initially identified at the level of title and abstract. And then the full-length articles were assessed further. Two authors checked the citations separately, a discussion was made to achieve consensus once controversies existed.

\section{Inclusion and exclusion criteria}

Only articles in English were included. The inclusion criteria were: (I) studies that reported the impact of RASi on mortality after SAVR; (II) studies that reported the impact of RASi on mortality after TAVR; (III) studies that illustrated the particular number of death or survival curve regarding the RASi after AVR. Studies were excluded if one of the following existed: (I) studies were case reports, reviews, abstracts, guidelines, editorials, comments and conference presentations; (II) studies that were not related to human.

\section{Data extraction and quality assessment}

Two independent authors ( $\mathrm{L}$ Zeng and J Li) extracted the 
data and reached a consensus on all items from eligible studies including author, publication year, research category, type of RASi, sort of AVR, follow-up time and mortality. If mortality or number of death was not presented directly, digitizing software (Engauge Digtizer 4.1) were utilized to acquire relevant data from survival curve (14).

Study quality was evaluated using the Cross-Sectional/ Prevalence Study Quality Assessment Form or NewcastleOttawa Quality Assessment Scale (NOS) accordingly $(15,16)$.

\section{Data synthesis and statistical analysis}

We combined data that reporting the impact of RASi on all-cause mortality and cardiac-cause mortality after AVR. Pooled odds ratios (OR) were gained using the Review Manage 5.3 software. Hazard ratios (HRs) were combined by using generic inverse variance method. Heterogeneity was evaluated by calculating the $\mathrm{I}^{2}$ statistic and its $\mathrm{P}$ value. Random-effects model was used when the $\mathrm{I}^{2}$ statistic was more than $50 \%$ and its $\mathrm{P}$ value was less than 0.05 , otherwise, the fixed-effects model was carried out. Two-sided $\mathrm{P}$ values of 0.05 were considered statistically significant.

\section{Publication bias analysis}

Publication bias was assessed by visual inspection of the symmetry of the funnel plot. However, if the number of pooled studies was small, publication bias was not performed.

\section{Subgroup analysis}

Several subgroup analyses were performed including the impact of RASi on all-cause mortality within 30 days, 1 year and beyond 1 year, the impact of RASi on cardiac mortality beyond 1 year. The effect of RASi after SAVR and TAVR were carried out respectively. And, the influence of RASi on all-cause mortality after AVR based on the baseline LVEF was also performed. Besides, the impact of ACEI versus $\mathrm{ARB}$ after AVR was also conducted.

\section{Results}

\section{Studies selection}

The process of study selection was presented in Figure 1.
A total of 126 potential relevant citations were identified after initial search from PubMed database. After careful review of the title and abstract, 114 studies were excluded. There were 9 (17-25) articles incorporating 33,063 patients were eligible after reading full text. A summary of included studies is illustrated in Table 1.

\section{Quality assessment}

The quality of included single-arm studies were assessed using Cross-Sectional/Prevalence Study Quality, while the quality of eligible cohort studies was evaluated by using the NOS scale. Overall quality of these included studies was good.

\section{Impact of RASi on all-cause mortality after AVR}

A total of $6(17,19,21,22,24,25), 6(18,19,21,22,24,25)$, $6(17-19,21,24,25)$ studies recruiting $27,435,22,864$ and 9,753 patients reported the effect of RASi after AVR, which revealed that patients taking RASi had a significant lower risk for mortality at 30 days (OR, $0.80,95 \% \mathrm{CI}, 0.69$ to 0.94, $\mathrm{I}^{2}=0 \%$, Figure 2), 1 year (OR, $0.75,95 \%$ CI, 0.69 to $0.81, \mathrm{I}^{2}=77 \%$, Figure 3) and beyond 1 year (OR, 0.52, 95\% CI, 0.38 to $0.73, \mathrm{I}^{2}=86 \%$, Figure 4) after AVR respectively, compared to those patients without RASi.

\section{Impact of RASi on all-cause mortality after TAVR}

Consistently, there were $4(17,21,22,25), 4(18,21,22,25)$, and $4(17,18,21,25)$ studies reporting that patients who took RASi had lower mortality at 30 days (OR, 0.76, 95\% CI, 0.63 to $0.92, \mathrm{I}^{2}=0 \%$, Figure 2 ), 1 year (OR, $0.76,95 \%$ CI, 0.70 to $0.95, \mathrm{I}^{2}=86 \%$, Figure 3) and beyond 1 year (OR, $0.65,95 \%$ CI, 0.45 to $0.94, \mathrm{I}^{2}=80 \%$, Figure 4 ) on the basis of incorporating $22,829,20,604$ and 7,493 patients separately.

\section{Impact of RASi on all-cause mortality after SAVR}

While, pooled studies displayed that patients having the description of RASi was associated with lower mortality at 1 -year (OR, $0.53,95 \% \mathrm{CI}, 0.29$ to $0.95, \mathrm{I}^{2}=41 \%$, Figure 3) and beyond 1-year (OR, 0.39, 95\% CI, 0.25 to $0.61, \mathrm{I}^{2}=73 \%$, Figure 4) mortality, but not at 30-day (OR, $0.90,95 \%$ CI, 0.68 to $1.19, \mathrm{I}^{2}=48 \%$, Figure 2), based on $2(19,24), 2(19,24)$ and $2(19,24)$ studies including 2,260 , 2,260 and 4,606 patients. 
Table 1 Characteristics of included studies

\begin{tabular}{|c|c|c|c|c|c|c|c|}
\hline Author & Year & Study design & Type of AVR & Number & Medication & Follow-up & Outcome \\
\hline Ochiai (18) & 2018 & Prospective cohort & TAVR & 560 & ACEI, ARB & 2 years & All-cause mortality \\
\hline Magne (19) & 2018 & Retrospective cohort & SAVR & 508 & ACEI, ARB & 8 years & All-cause mortality \\
\hline $\begin{array}{l}\text { Klinkhammer } \\
\text { (21) }\end{array}$ & 2019 & Retrospective cohort & TAVR & 169 & ACEI, ARB & 2 years & All-cause mortality \\
\hline Inohara (22) & 2018 & Retrospective cohort & TAVR & 21,312 & ACEI, ARB & 1 year & $\begin{array}{l}\text { All-cause mortality, } \\
\text { readmission for heart } \\
\text { failure }\end{array}$ \\
\hline Goel (24) & 2014 & Retrospective cohort & SAVR & 1,752 & ACEI, ARB & 5.8 years & All-cause mortality \\
\hline Chen (25) & 2019 & Prospective cohort & TAVR & 3,979 & ACEI, ARB & 2 years & $\begin{array}{l}\text { All-cause mortality, } \\
\text { cardiovascular mortality }\end{array}$ \\
\hline
\end{tabular}

TAVR, transcatheter aortic valve replacement; SAVR, surgical aortic valve replacement; ACEl, angiotensin-converting enzyme inhibitor; $\mathrm{ARB}$, angiotensin receptor blocker; ARNI, angiotensin receptor-neprilysin inhibitor.

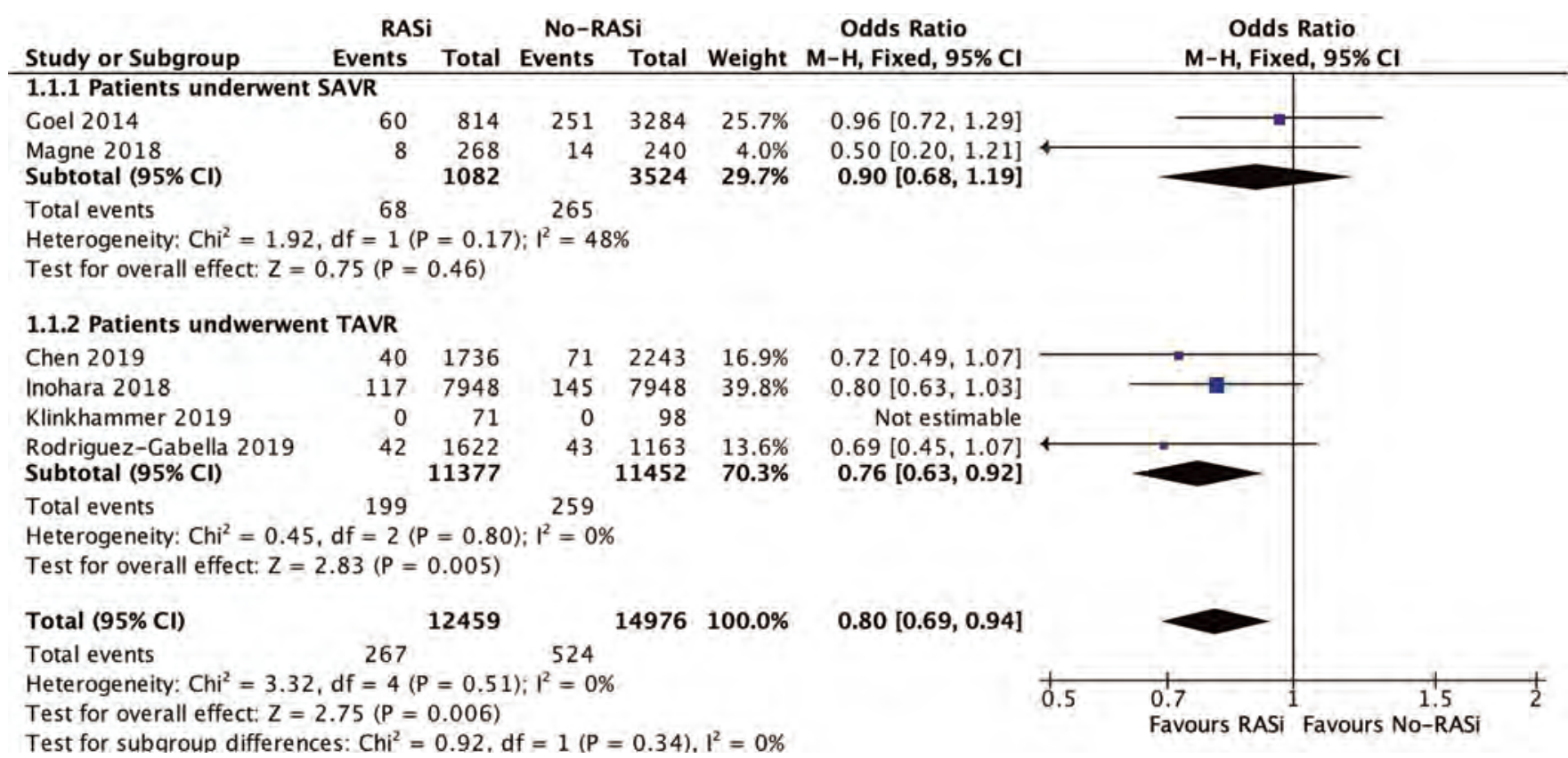

Figure 2 The impact of RASi on 30-day all-cause mortality after AVR. RASi, renin-angiotensin system inhibitor; SAVR, surgical aortic valve replacement; TAVR, transcatheter aortic valve replacement. 


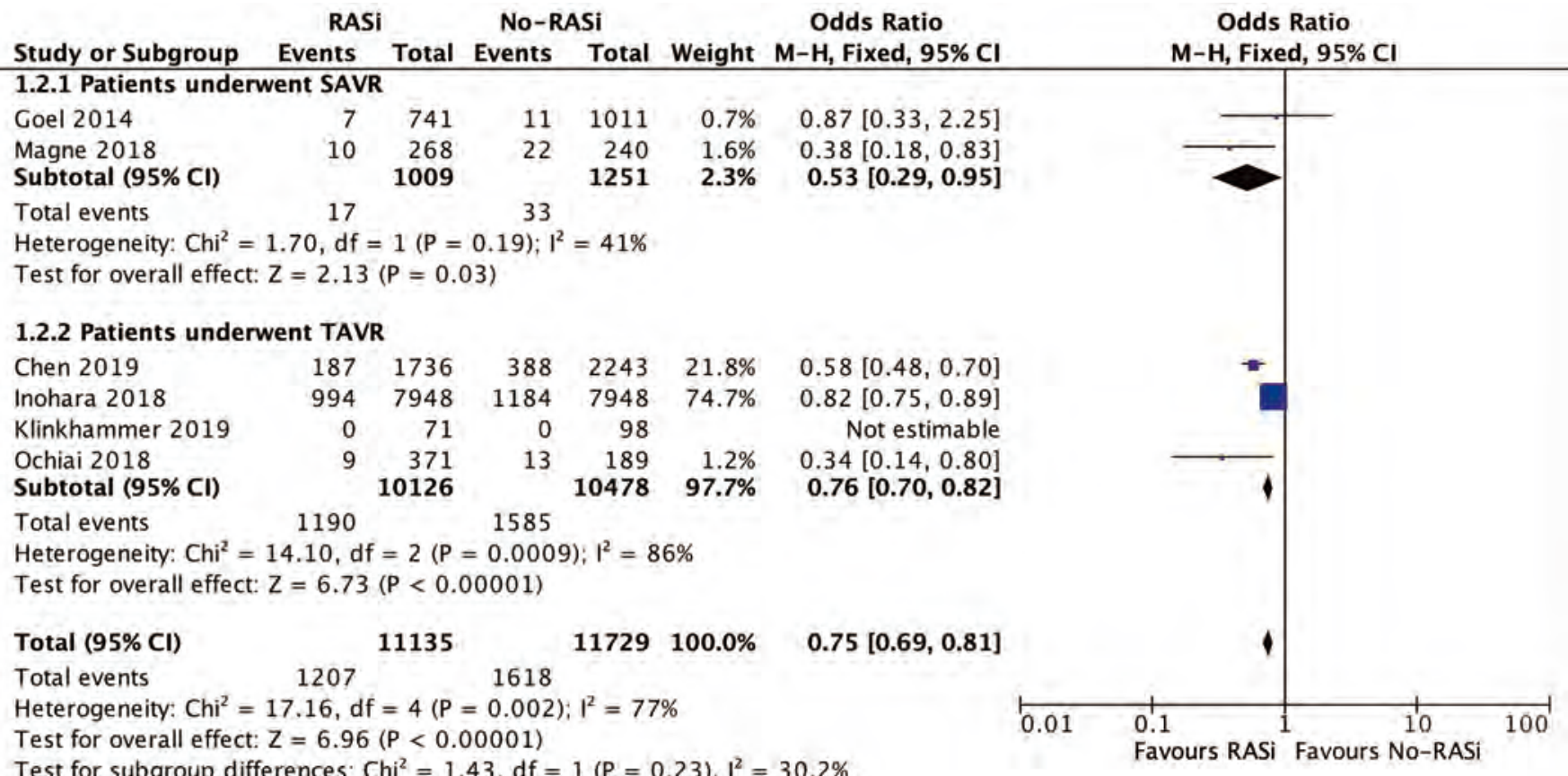

Figure 3 The impact of RASi on mortality within 1 year after AVR. RASi, renin-angiotensin system inhibitor; SAVR, surgical aortic valve replacement; TAVR, transcatheter aortic valve replacement.

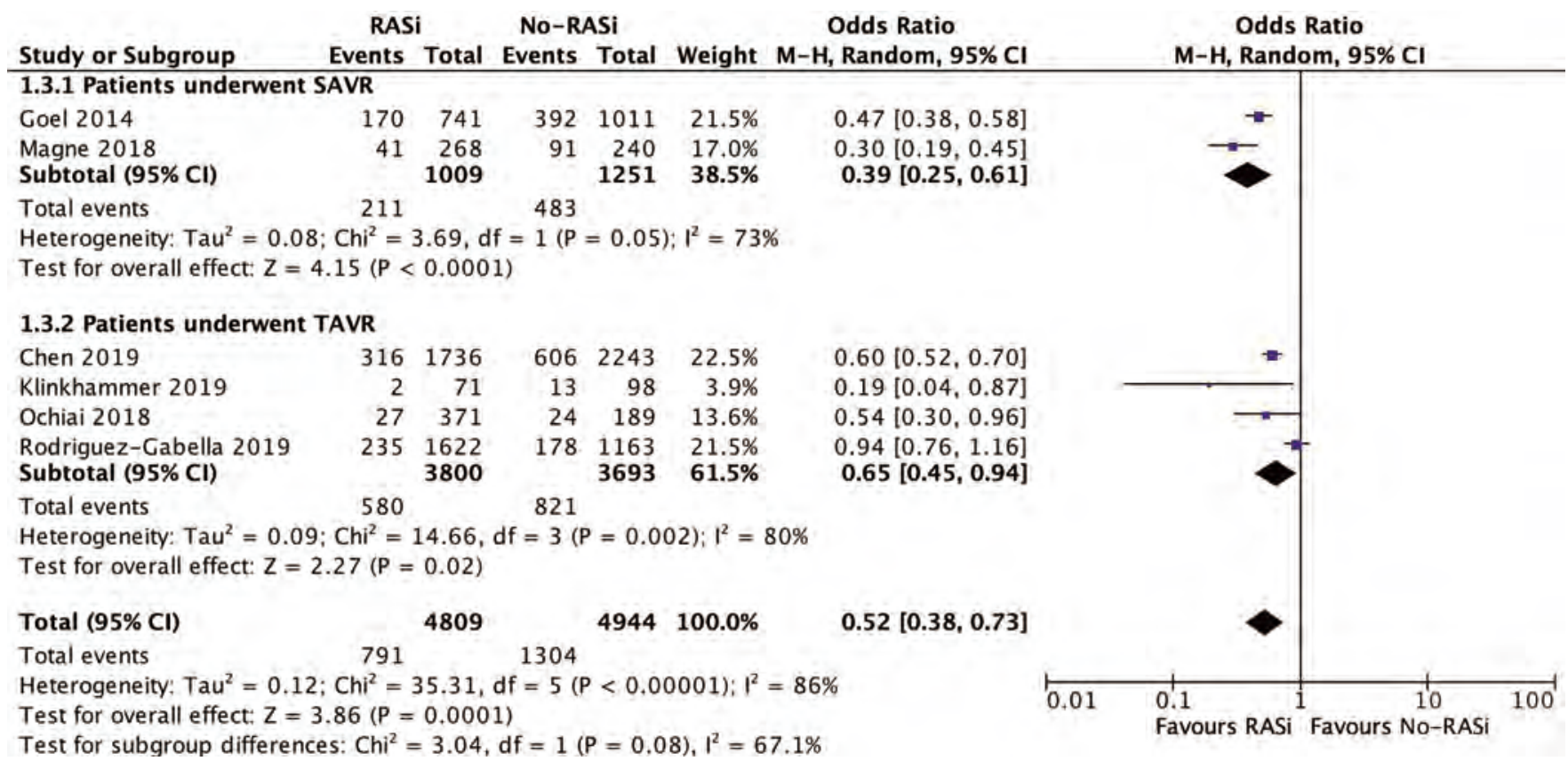

Figure 4 The impact of RASi on all-cause mortality beyond 1 year after AVR. RASi, renin-angiotensin system inhibitor; SAVR, surgical aortic valve replacement; TAVR, transcatheter aortic valve replacement. 


\section{Impact of RASi on all-cause mortality after AVR by using univariate and multivariate analysis}

In order to exclude the influence of confounders on outcome, we also pooled studies that reported the HR of RASi after AVR. We found that patients taking RASi was associated with reduced mortality beyond 1 year compared to those did not take RASi after AVR (HR, 0.84, 95\% CI, 0.77 to $0.91, \mathrm{I}^{2}=82 \%$, Figure S1) by using univariate analysis based on the combination of 7 (17-19,22-25) studies. In line with pooled univariate analysis, combined multivariate analysis also revealed a significant improvement of longterm survival in patients with RASi after AVR (HR, 0.87, $95 \%$ CI, 0.84 to $0.91, \mathrm{I}^{2}=0 \%$, Figure S2) on the basis of pooling $5(18-20,24,25)$ studies.

\section{Impact of RASi on all-cause mortality after TAVR by using univariate and multivariate analysis}

A total of $5(17,18,22,23,25)$ studies found that patients taking RASi had lower risk for mortality beyond 1 year than those patients without RASi after TAVR (HR, 0.89, 95\% CI, 0.82 to $0.96, \mathrm{I}^{2}=71 \%$, Figure S1) by using univariate analysis. Consistently, combined multivariate analysis also figured out that patients with RASi after TAVR were associated with significant reduced mortality beyond 1 year (HR, 0.87, $95 \%$ CI, 0.84 to $0.91, \mathrm{I}^{2}=0 \%$, Figure S2) by pooling $2(18,25)$ studies.

\section{Impact of RASi on all-cause mortality after SAVR by using univariate and multivariate analysis}

Except for the impact of RASi after TAVR, we also pooled studies that reported the impact of RASi after SAVR by using univariate and multivariate analysis. There were 2 $(19,24)$ and $3(19,20,24)$ studies displaying that patients taking RASi were associated lower mortality beyond 1 year compared to those patients without RASi after SAVR by using univariate analysis (HR, $0.73,95 \%$ CI, 0.56 to 0.96 , $\mathrm{I}^{2}=88 \%$, Figure $\mathrm{S} 1$ ) and multivariate analysis (HR, 0.89, $95 \%$ CI, 0.84 to $0.93, I^{2}=0 \%$, Figure S2) respectively.

\section{Impact of $R A S i$ on all-cause mortality regarding the baseline LVEF}

Combined results $(22,25)$ found that patients with reduced LVEF, taking RASi were associated with numerically lower mortality (HR, $0.91,95 \% \mathrm{CI}, 0.77$ to $1.07, \mathrm{I}^{2}=76 \%$,
Figure S3). While, interestingly, patients with preserved LVEF $(22,25)$, having a prescription of RASi had significant lower mortality (HR, $0.90,95 \% \mathrm{CI}, 0.87$ to $0.94, \mathrm{I}^{2}=0 \%$, Figure S3) than those patients without RASi. Besides, Goel et al. (24) also found that the favorable effect of RASi after SAVR was consistent across patients with reduced $(\mathrm{P}<0.05)$ and preserved LVEF $(\mathrm{P}<0.05)$.

\section{Impact of $R A S i$ on all-cause mortality regarding the type of RASi}

Magne et al. (19) described the relationship between the type of RASi and mortality, reporting that ARB had the best long-term survival, ACEI had the intermediate survival as compared with those patients without RASi (8-year survival, ARB vs. ACEI vs. no-RASi, $87 \%$ vs. $79 \%$ vs. $52 \%$, $\mathrm{P}<0.0001)$. Besides, after multivariable adjustment, they also demonstrated that patients with ARB was associated with lower risk for long-term all-cause mortality after SAVR (HR, $0.66,95 \%, 0.48$ to 0.93$)$, compared to those patients with ACEI.

We also pooled studies that reported the impact of ACEI and $\mathrm{ARB}$ on all-cause mortality beyond 1 year in patients undergoing TAVR. The combined results showed that both ARB $(22,25)$ (HR, $0.89,95 \%$ CI, 0.85 to $0.94, \mathrm{I}^{2}=0 \%$, Figure S4) and ACEI $(22,25)$ (HR, $0.89,95 \%$ CI, 0.81 to $0.99, \mathrm{I}^{2}=79 \%$, Figure S4) had lower all-cause mortality than those patients without RASi. Furthermore, there was no significant difference between patients taking ARB and ACEI after TAVR beyond 1 year $(22,25)$ (ARB $v$ s. ACEI, HR, $0.98,95 \%$ CI, 0.89 to $1.09, \mathrm{I}^{2}=62 \%$, Figure $\mathrm{S} 4$ ).

\section{Impact of RASi on cardiovascular mortality after TAVR}

In addition to all-cause mortality, we also performed analysis to investigate the effect of RASi on cardiovascular mortality after TAVR. Pooled studies demonstrated that patients with RASi had lower cardiovascular mortality than those patients without RASi after TAVR [30 days $(17,25)$, OR, 0.63, 95\% CI, 0.44 to $0.90, \mathrm{I}^{2}=22 \% ; 1$ year $(17,25)$, OR, $0.60,95 \% \mathrm{CI}$, 0.50 to $0.73, \mathrm{I}^{2}=0 \%$; beyond 1 year $(17,25), \mathrm{OR}, 0.63,95 \%$ CI, 0.54 to $0.74, I^{2}=0 \%$, Figure S5].

Consistently, pooled univariate $(17,25)$ ( HR, 0.83, $95 \%$ CI, 0.78 to $0.89, \mathrm{I}^{2}=0 \%$, Figure S6) and multivariate $(17,25)$ (HR, $0.84,95 \%$ CI, 0.78 to $0.91, \mathrm{I}^{2}=0 \%$, Figure S6) analyses showed that patients taking RASi after TAVR were correlated with reduced cardiovascular mortality beyond 1-year follow-up, compared to those patients who did not 
take RASi.

\section{Discussion}

The main results of the present study were as follows: (I) patients with RASi had lower all-cause mortality at 30 days (OR, $0.80,95 \%$ CI, 0.69 to 0.94 ), 1 year (OR, $0.75,95 \%$ CI, 0.69 to 0.81 ) and beyond 1 year (OR, $0.52,95 \%$ CI, 0.38 to 0.73 ) after AVR; (II) the combined results showed that both ARB (HR, 0.89, 95\% CI, 0.85 to 0.94 ) and ACEI (HR, $0.89,95 \%$ CI, 0.81 to 0.99 ) had lower all-cause mortality after TAVR, compared to those without RASi; (III) patients with preserved LVEF having a prescription of RASi were associated with reduced mortality (HR, 0.90, 95\% CI, 0.87 to 0.94 ) TAVR; (IV) pooled multivariable analysis also revealed that patients taking RASi after TAVR was associated with lower risk for cardiovascular mortality (HR, $0.84,95 \%$ CI, 0.78 to 0.91 ).

Myocardial hypertrophy was common in patients with AS, secondary to LV outflow obstruction and increased afterload (26). Progressive myocardial hypertrophy would yield fibrosis and subsequent LV dysfunction (26). More evidence found that RAS contributed to the occurrence of myocardial hypertrophy and fibrosis, RASi could reverse myocardial fibrosis to some extent (2). However, in the past, taking RASi in patients with AS was considered to be unsafe because the vasodilation effect in the presence of $\mathrm{LV}$ outflow obstruction would cause hypotension (5). In fact, patients with AS taking RASi was not only safe but also engendered favorable effect on survival, $\mathrm{LV}$ regression and even AS progression $(6,7)$.

Existing data reported that patients with LV hypertrophy and fibrosis undergoing AVR was associated with increased mortality, and irreversible LV hypertrophy as well as fibrosis after AVR was not rare which also affected long-term mortality $(10,11,13)$. However, no specific recommendations were provoked to take optimal medication following AVR to enhance the outcome of AVR including SAVR and TAVR. Therefore, there was still a gap regarding the optimal medication after AVR that could improve longterm prognosis.

Although several studies reported the impact of RASi on mortality after AVR, their results were controversy (18,23-25). The present study found that patients receiving RASi who underwent AVR were associated with reduced all-cause mortality at 30 days, 1 year and beyond 1 year, as compared with those patients without RASi, which is in consistence with previous propensity score-matched population studies $(17,22,25)$. The combined results were still positive after adjusting confounders independent of the type of RASi including ACEI and ARB. The beneficial effect of RASi on all-cause mortality may be attributed to the decreased cardiovascular mortality in patients with RASi as the present study also revealed that patients undergoing TAVR with RASi had lower cardiovascular mortality than those patients without RASi. This might be account for the potential role of RASi in further attenuation of LV hypertrophy and fibrosis after AVR, since incompletely reversed LV hypertrophy and fibrosis after AVR were correlated with increased mortality $(17,18,24,27)$.

Increasing evidence demonstrated the significant benefit of RASi in patients with reduced ejection fraction, while the impact of RASi on clinical outcome in patients with preserved ejection fraction was negative (28). However, we found that the beneficial effect of RASi after TAVR was independent of LVEF which is in line with prior study incorporating patients after SAVR described by Goel et al. (24). This might be related to the different pathophysiological mechanism for preserved ejection fraction in patients with AS and other cardiovascular diseases (29). A randomized clinical trial (RCT) containing 114 patients with preserved ejection fraction undergoing SAVR demonstrated that description of candesartan was associated with increased reverse LV remodeling as compared to those patients without RASi, which also might be suggestive of positive effect in patients with TAVR (27). Since the effect of RASi in patients following AVR primarily based on retrospective analysis, RCTs are warranted to evaluate the impact of RASi on mortality after AVR including SAVR and TAVR. Fortunately, RASTAVI, an ongoing RCT, could provide more information to determine the use of RASi (30).

\section{Limitation}

Firstly, all the included studies are not RCTs, which might have selection bias. Patients receiving RASi might be more stable and healthier that could tolerate the treatment of RASi. However, the present study found that patients having description of RASi was associated with reduced long-term mortality even after adjustment of confounders; Secondly, several combined results were based on fewer studies with considerable heterogeneity, which needed more studies to confirm these hypothesis-generating findings. 


\section{Conclusions}

Patients with RASi had reduced short-term and long-term all-cause mortality after AVR than those without RASi. Description of RASi was associated with lower risk for cardiovascular mortality in patients undergoing TAVR. Further studies were needed to confirm these findings.

\section{Acknowledgments}

We acknowledged Dr. JiaYu Tsauo for helping check and edit language.

Funding: This work was supported by Post-Doctor Research Project, West China Hospital, Sichuan University (2018HXBH083), National Natural Science Foundation of China (81900348, 81970325); Key Science and Technology Department of Sichuan Science and Technology Department (2019YFS0299); West China Hospital “1.3.5" Discipline of Excellence Project-Percutaneous aortic valve implantation.

\section{Footnote}

Reporting Checklist: The authors have completed the PRISMA reporting checklist. Available at http://dx.doi. org/10.21037/apm-20-1155

Conflicts of Interest: All authors have completed the ICMJE uniform disclosure form (available at http://dx.doi. org/10.21037/apm-20-1155). The authors have no conflicts of interest to declare

Ethical Statement: The authors are accountable for all aspects of the work in ensuring that questions related to the accuracy or integrity of any part of the work are appropriately investigated and resolved.

Open Access Statement: This is an Open Access article distributed in accordance with the Creative Commons Attribution-NonCommercial-NoDerivs 4.0 International License (CC BY-NC-ND 4.0), which permits the noncommercial replication and distribution of the article with the strict proviso that no changes or edits are made and the original work is properly cited (including links to both the formal publication through the relevant DOI and the license). See: https://creativecommons.org/licenses/by-nc$\mathrm{nd} / 4.0 /$.

\section{References}

1. Bing R, Cavalcante JL, Everett RJ, et al. Imaging and Impact of Myocardial Fibrosis in Aortic Stenosis. JACC Cardiovasc Imaging 2019;12:283-96.

2. Lindman BR, Clavel MA, Mathieu P, et al. Calcific aortic stenosis. Nat Rev Dis Primers 2016;2:16006.

3. Chang SM, Granger CB, Johansson PA, et al. Efficacy and safety of angiotensin receptor blockade are not modified by aspirin in patients with chronic heart failure: a cohort study from the Candesartan in Heart failure--Assessment of Reduction in Mortality and morbidity (CHARM) programme. Eur J Heart Fail 2010;12:738-45.

4. Wing LM, Reid CM, Ryan P, et al. A comparison of outcomes with angiotensin-converting--enzyme inhibitors and diuretics for hypertension in the elderly. $\mathrm{N}$ Engl J Med 2003;348:583-92.

5. Cox NL, Abdul-Hamid AR, Mulley GP. Aortic stenosis and ACE inhibitors. Lancet 1998;352:1392.

6. Bang CN, Greve AM, Kober L, et al. Renin-angiotensin system inhibition is not associated with increased sudden cardiac death, cardiovascular mortality or all-cause mortality in patients with aortic stenosis. Int J Cardiol 2014;175:492-8.

7. Andersson C, Abdulla J. Is the use of renin-angiotensin system inhibitors in patients with aortic valve stenosis safe and of prognostic benefit? A systematic review and meta-analysis. Eur Heart J Cardiovasc Pharmacother 2017;3:21-7.

8. Helske-Suihko S, Laine M, Lommi J, et al. Is blockade of the Renin-Angiotensin system able to reverse the structural and functional remodeling of the left ventricle in severe aortic stenosis?. J Cardiovasc Pharmacol 2015;65:233-40.

9. Goh SS, Sia CH, Ngiam NJ, et al. Effect of ReninAngiotensin Blockers on Left Ventricular Remodeling in Severe Aortic Stenosis. Am J Cardiol 2017;119:1839-45.

10. Herrmann S, Fries B, Salinger T, et al. Myocardial Fibrosis Predicts 10-Year Survival in Patients Undergoing Aortic Valve Replacement. Circ Cardiovasc Imaging 2018;11:e007131.

11. Sugiura A, Weber M, von Depka A, et al. Myocardial fibrosis in patients undergoing transcatheter aortic valve replacement: impact on left ventricular reverse remodeling and long-term outcome. EuroIntervention 2020;15:1417-23.

12. Une D, Mesana L, Chan V, et al. Clinical Impact of Changes in Left Ventricular Function After Aortic Valve 
Replacement: Analysis From 3112 Patients. Circulation 2015;132:741-7.

13. Beach JM, Mihaljevic T, Rajeswaran J, et al. Ventricular hypertrophy and left atrial dilatation persist and are associated with reduced survival after valve replacement for aortic stenosis. J Thorac Cardiovasc Surg 2014;147:362-369.e8.

14. Tierney JF, Stewart LA, Ghersi D, et al. Practical methods for incorporating summary time-to-event data into metaanalysis. Trials 2007;8:16.

15. Margulis AV, Pladevall M, Riera-Guardia N, et al. Quality assessment of observational studies in a drug-safety systematic review, comparison of two tools: the NewcastleOttawa Scale and the RTI item bank. Clin Epidemiol 2014;6:359-68.

16. Zeng X, Zhang Y, Kwong Joey SW, et al. The methodological quality assessment tools for preclinical and clinical studies, systematic review and meta-analysis, and clinical practice guideline: a systematic review. J Evid Based Med 2015;8:2-10.

17. Rodriguez-Gabella T, Catala P, Munoz-Garcia AJ, et al. Renin-Angiotensin System Inhibition Following Transcatheter Aortic Valve Replacement. J Am Coll Cardiol 2019;74:631-41.

18. Ochiai T, Saito S, Yamanaka F, et al. Renin-angiotensin system blockade therapy after transcatheter aortic valve implantation. Heart 2018;104:644-51.

19. Magne J, Guinot B, Le Guyader A, et al. Relation Between Renin-Angiotensin System Blockers and Survival Following Isolated Aortic Valve Replacement for Aortic Stenosis. Am J Cardiol 2018;121:455-60.

20. Lassnigg A, Hiesmayr M, Frantal S, et al. Longterm absolute and relative survival after aortic valve replacement: a prospective cohort study. Eur J Anaesthesiol 2013;30:695-703.

21. Klinkhammer B. Renin-angiotensin system blockade after transcatheter aortic valve replacement (TAVR) improves intermediate survival. J Cardiovasc Thorac Res 2019;11:176-81.

Cite this article as: Zeng L, Li J, Yang J, Liao Y, Chen M. Impact of renin-angiotensin system blocker after aortic valve replacement-a systematic review and meta-analysis. Ann Palliat Med 2021;10(2):1244-1252. doi: 10.21037/apm-20-1155
22. Inohara T, Manandhar P, Kosinski AS, et al. Association of Renin-Angiotensin Inhibitor Treatment With Mortality and Heart Failure Readmission in Patients With Transcatheter Aortic Valve Replacement. JAMA 2018;320:2231-41.

23. Herron R, Nanjundappa A, Annie FH, et al. Our Experience with Transcatheter Aortic Valve Replacement Medication Outcomes from 2013 to 2016. Cureus 2019;11:e6030.

24. Goel SS, Aksoy O, Gupta S, et al. Renin-angiotensin system blockade therapy after surgical aortic valve replacement for severe aortic stenosis: a cohort study. Ann Intern Med 2014;161:699-710.

25. Chen S, Redfors B, Nazif T, et al. Impact of reninangiotensin system inhibitors on clinical outcomes in patients with severe aortic stenosis undergoing transcatheter aortic valve replacement: an analysis of from the PARTNER 2 trial and registries. Eur Heart J 2020;41:943-54.

26. Rader F, Sachdev E, Arsanjani R, et al. Left ventricular hypertrophy in valvular aortic stenosis: mechanisms and clinical implications. Am J Med 2015;128:344-52 .

27. Dahl JS, Videbaek L, Poulsen MK, et al. Effect of candesartan treatment on left ventricular remodeling after aortic valve replacement for aortic stenosis. Am J Cardiol 2010;106:713-9.

28. Agarwal V, Briasoulis A, Messerli FH. Effects of renin-angiotensin system blockade on mortality and hospitalization in heart failure with preserved ejection fraction. Heart Fail Rev 2013;18:429-37.

29. Shah SJ, Katz DH, Selvaraj S, et al. Phenomapping for novel classification of heart failure with preserved ejection fraction. Circulation 2015;131:269-79.

30. Amat-Santos IJ, Catalá P, Diez Del Hoyo F, et al. Impact of renin-angiotensin system inhibitors on clinical outcomes and ventricular remodelling after transcatheter aortic valve implantation: rationale and design of the RASTAVI randomised multicentre study. BMJ Open 2018;8:e020255. 


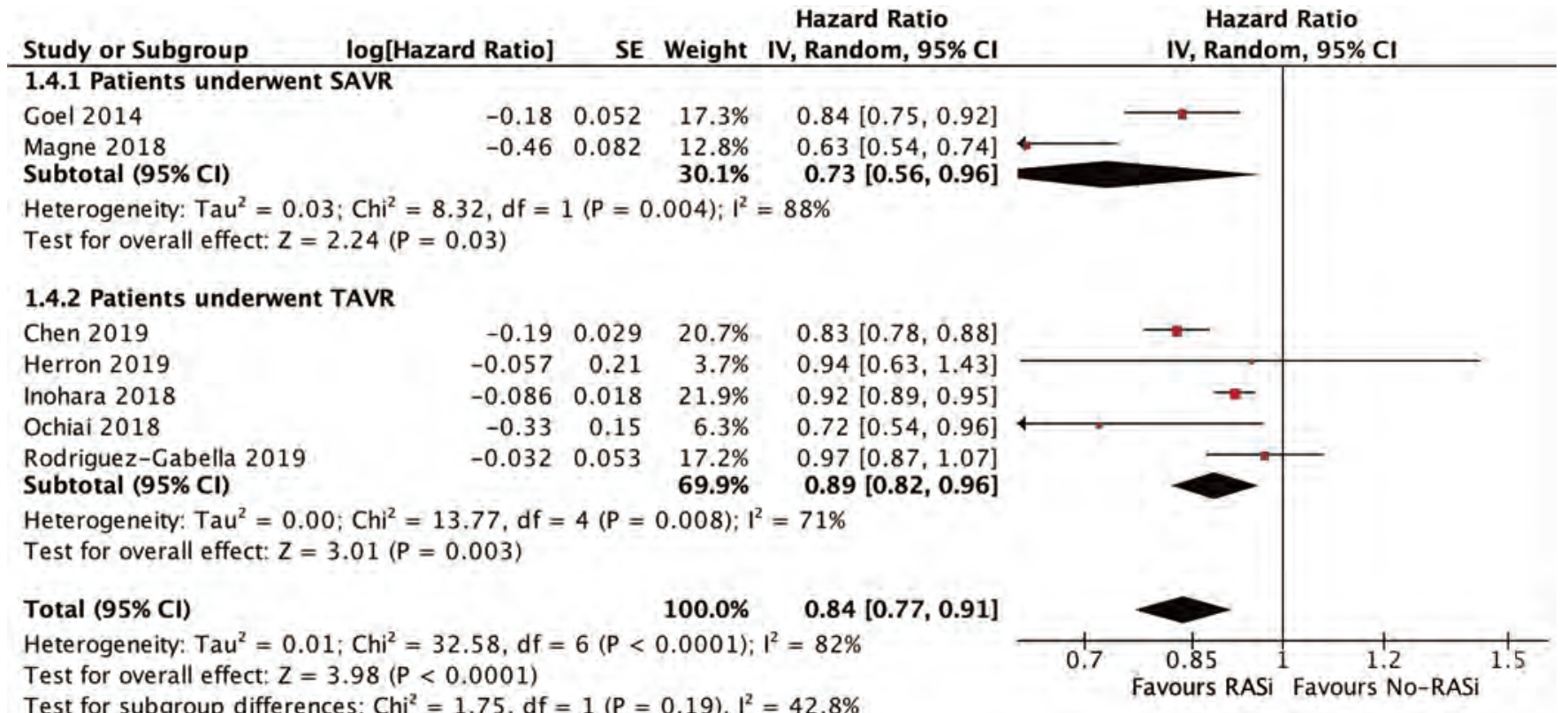

Figure S1 The impact of RASi on all-cause mortality beyond 1 year after AVR by pooling univariate estimate effects. RASi, reninangiotensin system inhibitor; SAVR, surgical aortic valve replacement; TAVR, transcatheter aortic valve replacement.

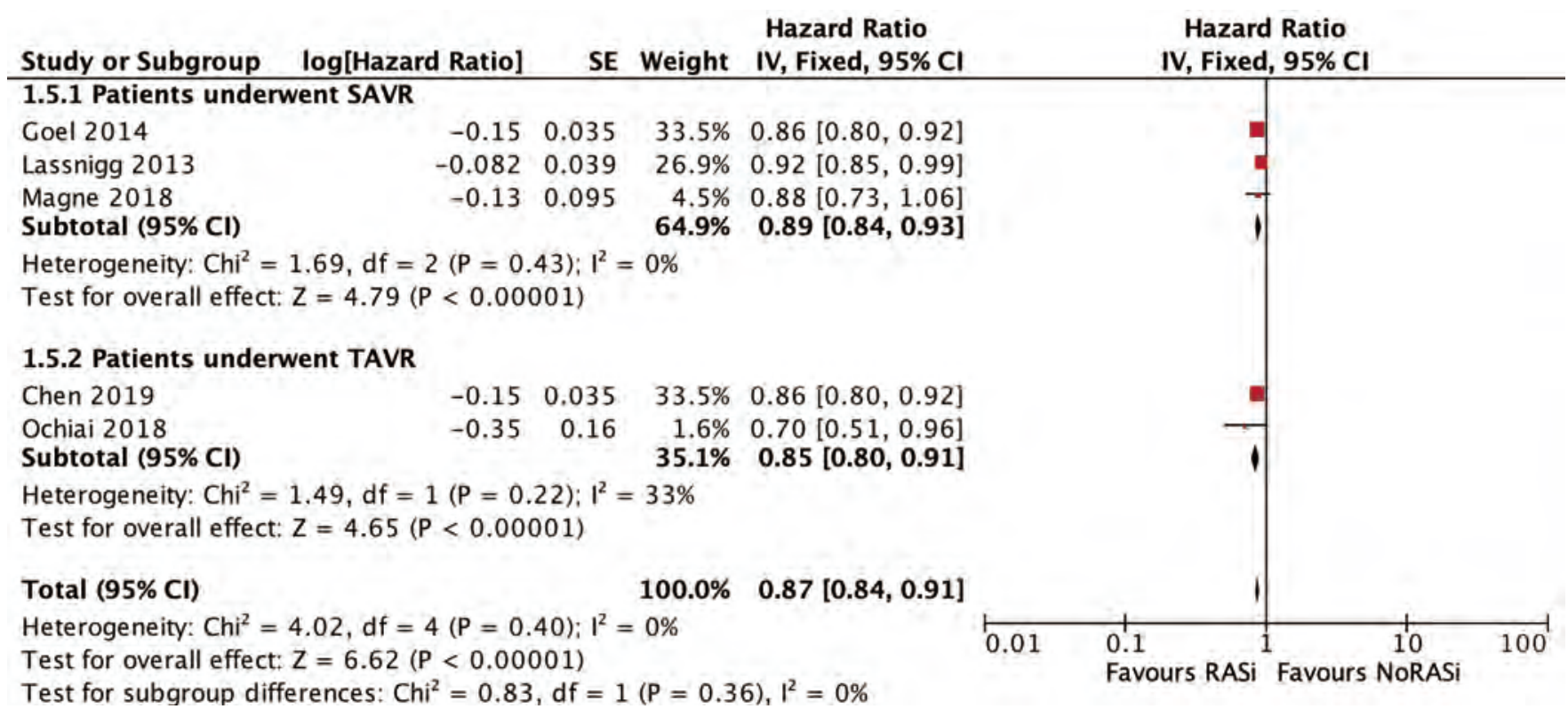

Figure S2 The impact of RASi on all-cause mortality beyond 1 year after AVR by pooling multivariate estimate effects. RASi, reninangiotensin system inhibitor; SAVR, surgical aortic valve replacement; TAVR, transcatheter aortic valve replacement. 


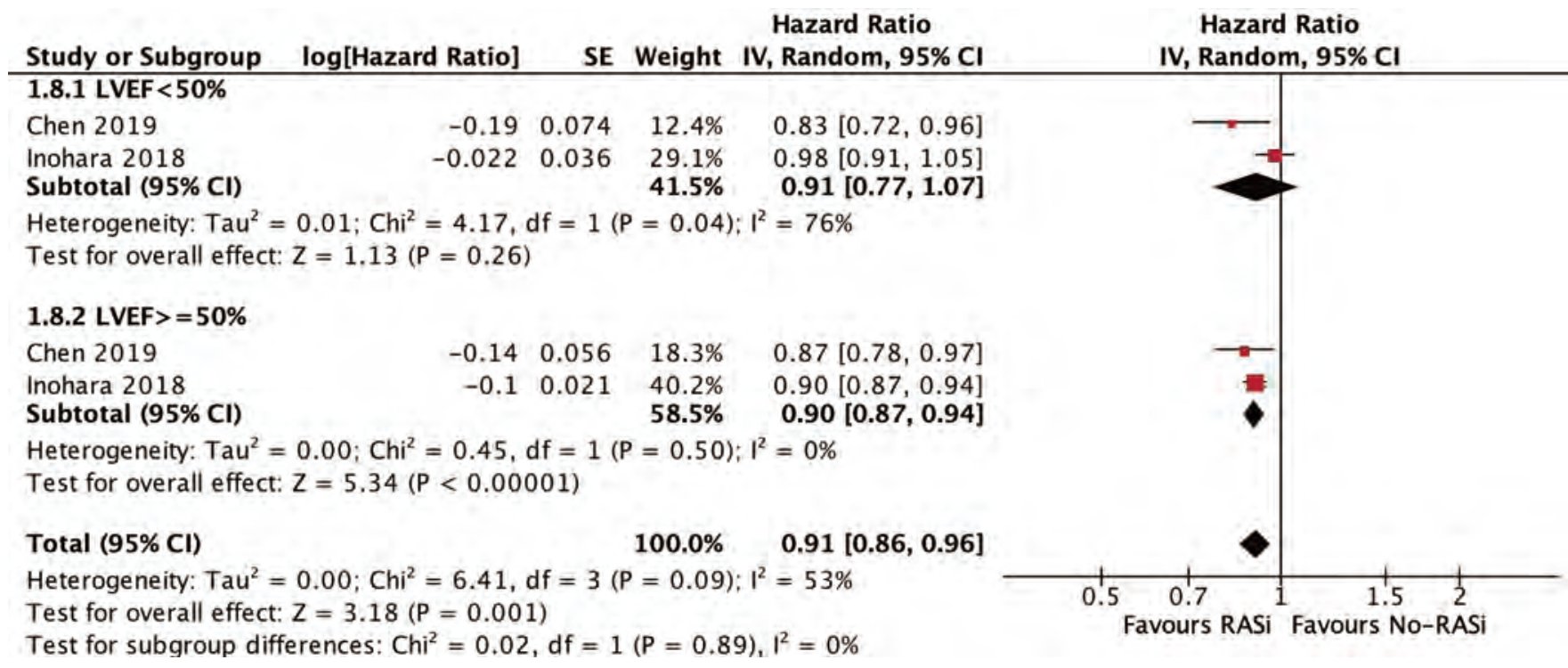

Figure S3 The impact of RASi on all-cause mortality after TAVR regarding the baseline left ventricular ejection fraction. RASi, reninangiotensin system inhibitor; TAVR, transcatheter aortic valve replacement; LVEF, left ventricular ejection fraction.

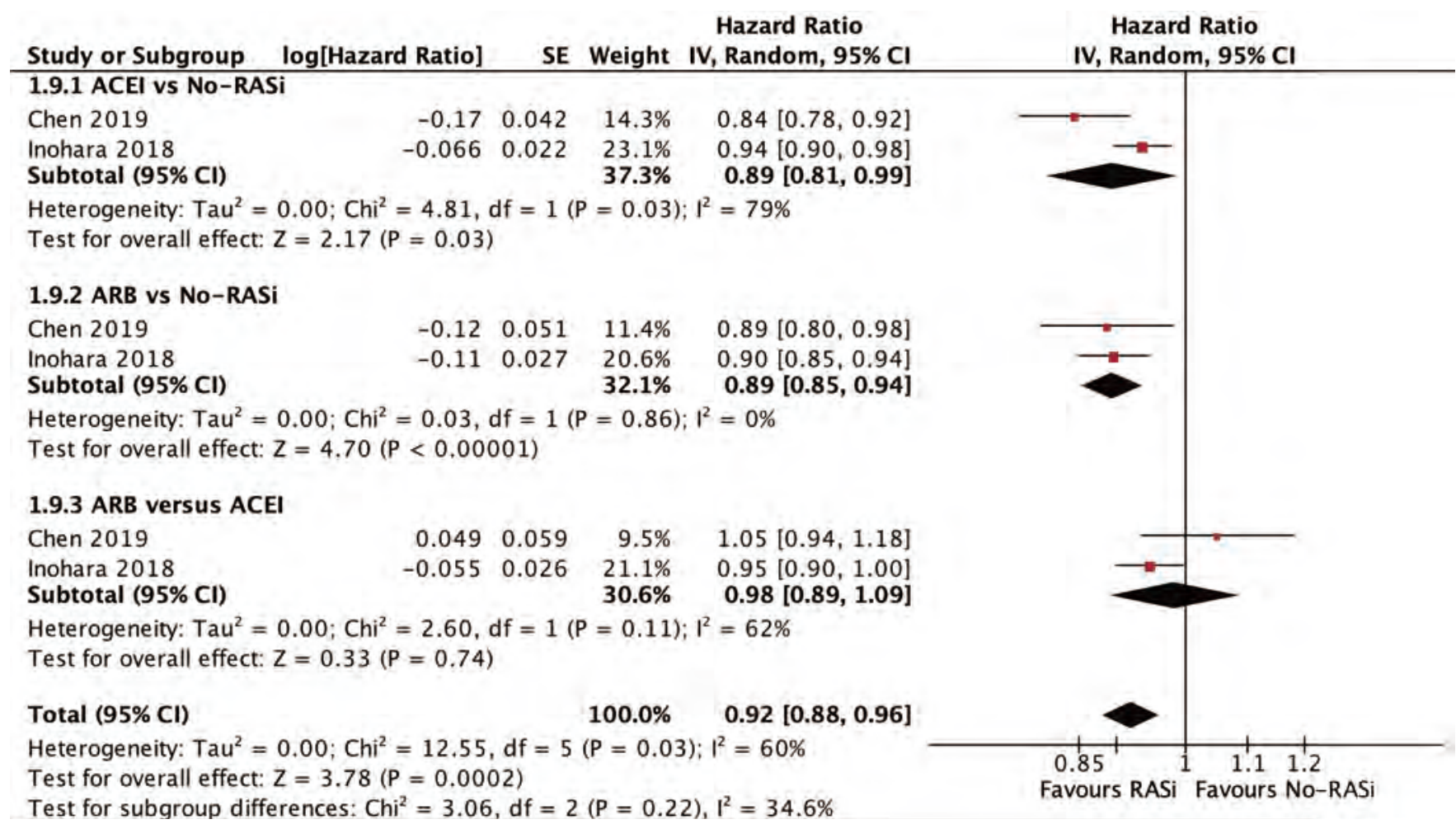

Figure S4 The impact of RASi on all-cause mortality after TAVR regarding the type of RASi. RASi, renin-angiotensin system inhibitor; TAVR, transcatheter aortic valve replacement; ACEI, angiotensin-converting enzyme inhibitor; ARB, angiotensin receptor blocker. 


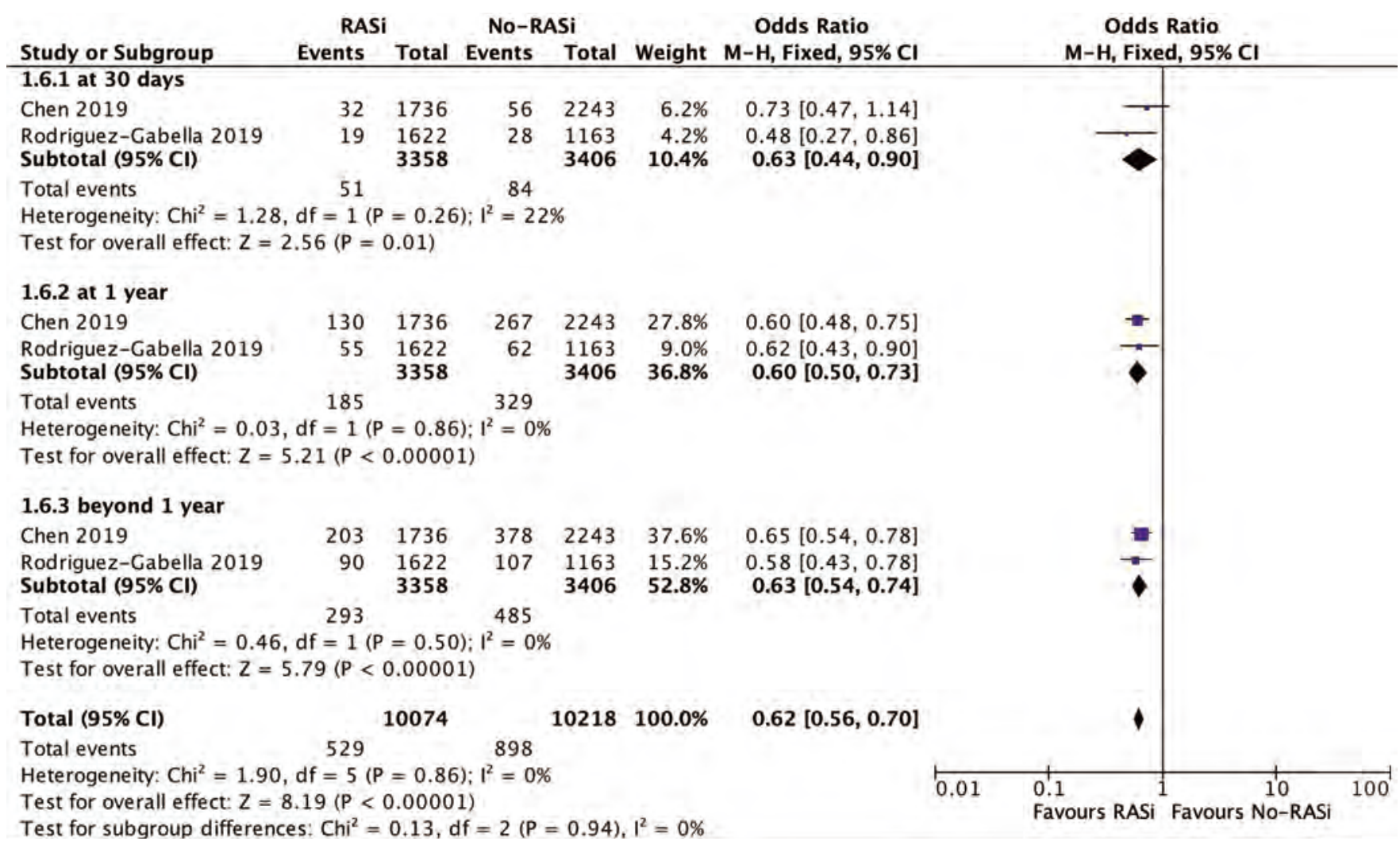

Figure S5 The impact of RASi on cardiovascular mortality after TAVR. RASi, renin-angiotensin system inhibitor; TAVR, transcatheter aortic valve replacement.

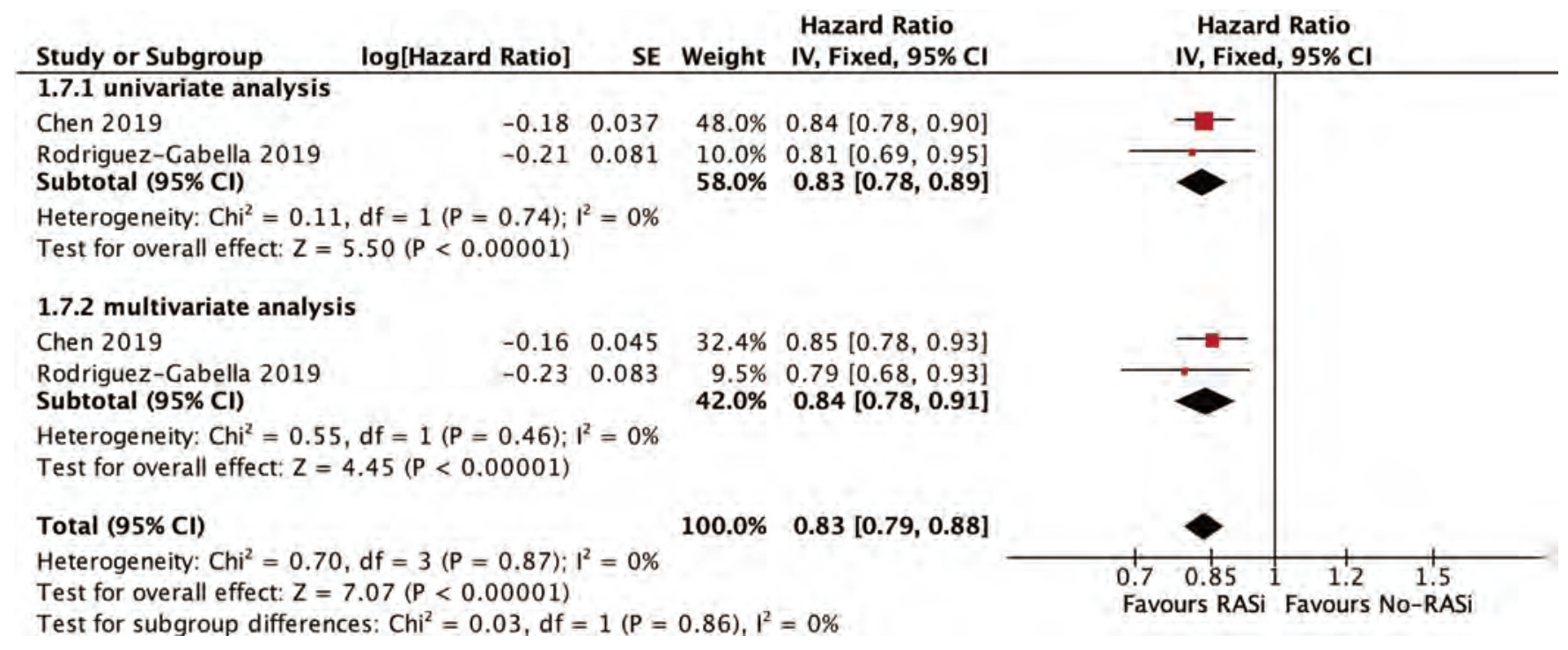

Figure S6 The impact of RASi on cardiovascular mortality beyond 1 year after TAVR by pooling univariate and multivariate estimate effects. RASi, renin-angiotensin system inhibitor; TAVR, transcatheter aortic valve replacement. 